Board of Governors of the Federal Reserve System

International Finance Discussion Papers

Number 695

December 2000

\title{
PREDICTABLE UNCERTAINTY IN ECONOMIC FORECASTING
}

Neil R. Ericsson

NOTE: International Finance Discussion Papers are preliminary materials circulated to stimulate discussion and critical comment. References to International Finance Discussion Papers (other than an acknowledgment that the writer has had access to unpublished material) should be cleared with the author or authors. Recent IFDPs are available on the Web at www.federalreserve.gov/pubs/ifdp/. 


\title{
PREDICTABLE UNCERTAINTY IN ECONOMIC FORECASTING
}

\author{
Neil R. Ericsson*
}

Abstract: This paper provides an introduction to predictable forecast uncertainty in empirical economic modelling. The sources of both predictable and unpredictable forecast uncertainty are categorized. Key features of predictable forecast uncertainty are illustrated by several analytical models, including static and dynamic models, and single-equation and multiple-equation models. Empirical models of the U.S. trade account, U.K. inflation, and U.K. real national income help clarify the issues involved.

Keywords: econometrics, economics, forecasting, models, uncertainty.

JEL classifications: C1, C53.

\footnotetext{
${ }^{*}$ Chapter for Companion to Economic Forecasting, Michael P. Clements and David F. Hendry (eds.), Blackwell Publishers, forthcoming. The author is a staff economist in the Division of International Finance, Board of Governors of the Federal Reserve System, Washington, D.C. 20551 U.S.A., and may be reached on the Internet at ericsson@frb.gov. The views in this paper are solely the responsibility of the author and should not be interpreted as reflecting the views of the Board of Governors of the Federal Reserve System or of any other person associated with the Federal Reserve System. I am grateful to Julia Campos, Mike Clements, Clive Granger, David Hendry, Jaime Marquez, and Hayden Smith for helpful discussions and comments; to Jurgen Doornik for providing me with a beta-test copy of GiveWin Version 2.00; and to the Bank of England for permission to reprint the fan chart and density function in Figures 4 and 5. All numerical results were obtained with PcGive Professional Version 9: see Doornik and Hendry (1996). Data and output listings for this paper's empirical results are available from the author and at www.federalreserve.gov/pubs/ifdp/2000/695/default.htm on the WorldWide Web.
} 


\section{Introduction}

Forecasts of economic variables play a prominent role in business decision-making, government policy analysis, and economic research. Forecasts often are model-based, with forecasts from an estimated model being constructed as the model's fitted values over a sample not used in estimation. Forecasts typically differ from the realized outcomes, with discrepancies between forecasts and outcomes reflecting forecast uncertainty. Depending upon the degree of forecast uncertainty, forecasts may range from being highly informative to utterly useless for the tasks at hand.

Measures of forecast uncertainty have numerous uses in economic practice. For instance, prior to the realization of outcomes, a measure of forecast uncertainty provides an assessment of the "expected" or predicted uncertainty of the forecast errors, helping to qualify the forecasts themselves and to give a picture of the expected range of likely outcomes. That is, a measure of forecast uncertainty helps distinguish between numerical accuracy and statistical accuracy in forecasts. Information about forecast uncertainty is important in addition to the forecast itself. Also, once outcomes are known, the corresponding forecast errors and the anticipated forecast uncertainty can be used to evaluate the models from which the forecasts were generated.

Thus, this paper considers forecast uncertainty in econometric modelling, analyzing at a general level the sources of uncertainty present in economic forecasting. At the outset, a distinction is made between predictable uncertainty and unpredictable uncertainty, with the focus of the paper being on the former. Key features of predictable forecast uncertainty are illustrated by several analytical models, including static and dynamic models, and single-equation and multiple-equation models. Empirical models of the U.S. trade account, U.K. inflation, and U.K. real national income clarify the issues involved.

This paper is organized as follows. Section 2 reviews a taxonomy for the sources of forecast uncertainty, partitioning those sources into ones that generate predictable uncertainty and ones that generate unpredictable uncertainty. This section then sketches a framework for analyzing economic forecasts. Combined, the taxonomy and the framework guide the choice of models examined in subsequent sections. Sections 3 and 4 analyze the properties of predictable uncertainty associated with forecasts from single-equation and multiple-equation models respectively. These sections highlight how predictable forecast uncertainty is affected by the forecast horizon and by the type of forecast model - whether static or dynamic, and whether a single equation

or a system. Section 5 considers various uses of predictable forecast uncertainty in economic modelling. Section 6 concludes.

Some preliminary comments will aid following the presentation below. This paper presupposes an understanding of "how economists forecast" on the level of the discussions in Granger (1989) and Hendry and Ericsson (2001). For the most part, the current paper restricts itself to time series and econometric models as the tools 
Table 1. A Taxonomy of the Sources of Model-based Forecast Error

1. Sources of predictable uncertainty

"what we know that we don't know"

(a) cumulation of future errors ("shocks") to the economy

(b) inaccuracies in estimates of the forecast model's parameters

2. Sources of unpredictable uncertainty

"what we don't know that we don't know"

(a) currently unknown future changes in the economy's structure

(b) mis-specification of the forecast model

(c) mis-measurement of the base-period data

for forecasting itself, where these models are assumed to be well-specified. Clements and Hendry (1998), Clements and Hendry (1999), and Ericsson and Marquez (1998) consider some of the generalizations required and implications for situations in which the empirical forecast model is mis-specified and for which that mis-specification is important. Wallis (1999b) inter alia discusses various ways of characterizing forecast uncertainty in macroeconomic modelling. Finally — and at a very practical level - figures, which are central to the paper's examples, typically appear as panels of graphs, with each graph designated by a suffix $a, b, c, \ldots$, row by row.

\section{Model-based Forecasts and Forecast Errors}

This section summarizes a taxonomy of forecast errors (Section 2.1) and a framework for analyzing economic forecasts (Section 2.2). Together, these set the stage for discussing predictable uncertainty in economic forecasting (Sections 3, 4, and 5).

\subsection{A Taxonomy of Forecast Uncertainty}

This subsection examines the determinants of forecast uncertainty, drawing on a taxonomy for the sources of model-based forecast error in Clements and Hendry (1998, Chapter 7.3, especially Table 7.1). ${ }^{1}$ Table 1 summarizes Clements and Hendry's taxonomy, partitioning the sources into "what we know that we don't know" (Items 1(a)$1(\mathrm{~b})$ ) and "what we don't know that we don't know" (Items 2(a)-2(c)), to paraphrase Maxine Singer (1997, p. 38). In practice, all of the listed sources are important when analyzing forecast uncertainty.

\footnotetext{
${ }^{1}$ Strictly speaking, "forecast uncertainty" should be called "forecast error uncertainty", as the forecast error is what is uncertain, not the forecast. However, following common usage in the literature, and for brevity's sake, the phrase "forecast uncertainty" is used throughout this paper.
} 
Items $1(\mathrm{a})$ and $1(\mathrm{~b})$ are predictable in the sense that the degree of uncertainty arising from them can be anticipated and even calculated. Item 1(a) - the cumulation of future shocks to the economy - captures the uncertainty inherent to future events. It contains shocks that would be expected to occur, given the model used in forecasting. Item $1(\mathrm{~b})$ results in "estimation uncertainty", which is due to using coefficient estimates in forecasting, rather than the underlying parameter values.

By contrast, Items 2(a), 2(b), and 2(c) are unpredictable and unanticipated. If their extent and nature were known, they could be incorporated into the model and they — or at least the uncertainty that they create - would be predictable and predicted. Interactions between the three sources of unpredictable uncertainty can be particularly important; see Clements and Hendry (1998, 1999). The current paper focuses on the two sources of predictable uncertainty, and primarily on inherent uncertainty - Item 1(a). That said, the sources of unpredictable uncertainty are central to evaluating empirical models; see Section 5 below.

At a more prosaic level, forecast uncertainty depends upon the variable being forecast, the type of model used for forecasting, the information available for constructing forecasts, and the economic process actually determining the variable being forecast. On the first, some variables may be inherently more difficult to forecast than others. For instance, imports and exports each might be highly predictable, and good models might exist for forecasting them. The trade balance - that is, the value of exports minus imports - might be quite difficult to forecast. In particular, by being the difference between two relatively large quantities (exports and imports), the trade balance is itself a relatively small quantity, whereas its forecast error reflects the forecast errors of both imports and exports. As another example, forecasting the level of the exchange rate might be relatively easy, in that the exchange rate in (say) a month's time is likely to be close to today's exchange rate. That said, forecasting the change in the exchange rate over the next month could be quite difficult. So, the particular variables being forecast and the transformations applied to those variables can affect the degree of forecast uncertainty present.

Secondly, forecast uncertainty depends upon the model that is being used for forecasting. Some models may simply be better for forecasting than others. Also, the particular form of the model determines what the predictable forecast uncertainty is, as distinct from the actual forecast uncertainty that arises. As Table 1 clarifies, that distinction exists because a model is a simplified characterization of the economy, not a reproduction of the economy. Sometimes that characterization is a good one, and sometimes it is not.

Thirdly, forecast uncertainty depends upon the information available for constructing the forecasts. This aspect is closely tied to the design of the forecast model. More information would seem to be beneficial for forecasting, and it is so in some situations. That said, when the model is mis-specified and there are structural breaks in the data, use of additional information can actually increase forecast uncertainty; 
Table 2. Some Issues in Designing Economic Forecasts

\begin{tabular}{ll}
\hline \hline $\begin{array}{l}\text { Relationship } \\
\text { of Interest, and DGP }\end{array}$ & $\begin{array}{l}\text { Characteristics } \\
\text { of the Forecast }\end{array}$ \\
\hline $\begin{array}{l}\text { Dimension } \\
\text { single equation }\end{array}$ & Dimension \\
multiple equations & scalar \\
vector \\
subsystem & Forecasts examined \\
full system & subset \\
Temporal form & full set \\
static & Forecast horizon \\
dynamic & one-step ahead \\
Distributions & multi-step ahead \\
error & Distributional property \\
coefficient estimates & mean (and bias) \\
initial conditions & variance (and MSFE) \\
\hline
\end{tabular}

see Clements and Hendry (1999, Chapter 2).

Fourthly, the underlying process generating the data plays a role in determining forecast uncertainty, such as by placing limits on the minimum forecast uncertainty obtainable from a model. That distinguishes between the predictable forecast uncertainty - that is, the forecast uncertainty anticipated, given the model — and the actual forecast uncertainty, which is the uncertainty arising from the combination of the model with the actual behavior of the economic data.

To consider the role of these and other aspects of forecasts per se, the next subsection draws on Ericsson and Marquez's (1998) framework for economic forecasting.

\subsection{A Framework for Interpreting Forecasts}

Ericsson and Marquez (1998) divide the mechanics of forecasting into three parts:

1. design, in which the characteristics of the forecasts and the relationship(s) of interest are specified;

2. evaluation, wherein the forecasts and their characteristics are calculated; and

3. post-evaluation analysis, in which the forecasts are presented.

The current paper employs all three aspects. That said, design is most relevant to the pedagogical approach taken in subsequent sections because forecast design specifies the relationship of interest for generating the forecasts and the characteristics of the forecasts being examined. Table 2 lists some details of forecast design. 
Table 3. A Guide to the Models Examined

\begin{tabular}{lll}
\hline \hline Model class (relationship of interest) & Model \\
\hline static single equation & $\mathrm{M}_{1}: \quad y_{t}=b z_{t}+u_{t}$ \\
dynamic single equation & $\mathrm{M}_{2}: \quad y_{t}=b y_{t-1}+u_{t}$ \\
(generalized) dynamic single equation & $\mathrm{M}_{3}: \quad y_{t}=b_{1} y_{t-1}+b_{2} z_{t}+u_{t}$ \\
simple dynamic system & $\mathrm{M}_{4}:\left\{\begin{array}{l}y_{t}=b z_{t}+u_{t} \\
z_{t}=c y_{t-1}+v_{t} \\
\text { (generalized) dynamic system }\end{array}\right.$ & $\mathrm{M}_{5}: \quad \begin{array}{r}\mathbf{x}_{t}=\mathbf{B} \mathbf{x}_{t-1}+\mathbf{w}_{t} \\
\end{array}$ \\
\hline
\end{tabular}

Design itself is subject to a tripartite division: the relationship of interest, the data generation process (DGP), and characteristics of the forecast. The relationship of interest may be classified by its dimension (single equation versus multiple equations), completeness (subsystem versus full system), temporal form (static versus dynamic), and distributional assumptions (for the error, for the coefficient estimates, and for the initial conditions). The underlying DGP may also be described in a similar fashion. The characteristics of the forecast include its dimension (scalar versus vector), the set of forecasts examined (subset versus full set), the forecast horizon (one-step ahead versus multiple-steps ahead), and the distributional property of interest (such as the forecast's mean, bias, variance, and mean square forecast error). These characteristics roughly parallel those for the relationship of interest and the DGP. However, even for similar categories, the classifications need not match: e.g., a relationship might be a multiple-equation complete system, while the forecast of interest is scalar.

Each combination of characteristics for the relationship of interest, the DGP, and the forecasts may imply different properties for the associated predictable uncertainty. Even the limited classification in Table 2 entails 13, $824\left(=\left(2^{3} \cdot 3\right)^{3}\right)$ possible combinations. Extensive analysis of these combinations is not feasible, nor is it necessary. Rather, a small set of combinations serves to highlight essential interactions between the various characteristics. Table 3 lists the five models examined in Sections 3 and 4 below: three single-equation models (one static, one dynamic, and one mixed), and two dynamic systems (one very specific, and the other general). Three issues listed in Table 2 (dimension, completeness, and temporal form) characterize these models.

Evaluation is the process of generating forecasts and calculating their characteristics, such as their variances. For some models, evaluation is analytic. However, even for relatively simple models, exact analytical results are not available, so calculation of forecasts and their characteristics may involve numerical integration, analytical approximations, Monte Carlo simulation, or bootstrapping. See Ericsson and Marquez (1998) for a summary. 
Post-evaluation analysis concerns the presentation of the forecasts and their characteristics, once calculated. Tables and graphs are common modes - graphs are used extensively below. Sometimes, the properties of forecasts are summarized through a statistic, as in the Chow (1960) statistic and the forecast-encompassing statistic; see Chong and Hendry (1986) on the latter. The purposes of the particular forecasting endeavor often govern the structure of post-evaluation analysis.

Before proceeding, a few comments are in order. First, the models considered are kept very simple for expositional reasons. Even so, many features of their forecasts' predictable uncertainty are characteristic of much more general models. Second, and relatedly, analytical techniques exist for examining forecast uncertainty from general linear models, and numerical techniques are available for nonlinear models if analytical solutions are unknown. Third, in order to simplify the analysis of predictable uncertainty, the econometric model underlying the relationship of interest is typically assumed to be the DGP. That said, Section 5 necessarily distinguishes between the DGP and the model when interpreting tests for the latter's constancy. Finally, the taxonomy in Table 1 and the framework above delineate the models in Table 3 and their forecasts' properties, which are the focus of the remaining sections.

\section{Predictable Uncertainty for Static and Dynamic Single-equation Models}

This section calculates analytically and numerically the predictable forecast uncertainty for some specific static and dynamic single-equation models. The aim is to characterize how various features listed in Table 2 affect predictable forecast uncertainty. Thus, this section focuses on the effects of static versus dynamic specifications, of forecast horizon, of parameter values, and of estimation uncertainty. The subsequent section (Section 4) examines forecasting from a system of equations. Straightforward results follow from simple assumptions, such as univariate or bivariate DGPs, single-equation or two-equation models, first-order dynamics (at most), stationarity, and ergodicity. Many — but not all — results carry through or generalize naturally under weaker restrictions.

Section 3.1 considers a simple static model, Section 3.2 a simple dynamic model, and Section 3.3 compares and contrasts these results, with further evidence from an application to forecasting U.K. net national income. Each example proceeds pedagogically with the specification of the model, the forecasts, the forecast errors, and the forecast errors' properties. 


\subsection{A Static Single Equation}

This subsection describes the model, model-based forecasts, forecast errors, and their properties for a simple static single-equation model. This model serves as a benchmark for comparison with other models, and it creates a template for the analysis of other models. Chow (1960) discusses forecasts from this model in detail.

This first model (denoted $\mathrm{M}_{1}$ ) is a static single equation:

$$
\mathrm{M}_{1}: \quad y_{t}=b z_{t}+u_{t} \quad u_{t} \sim \mathrm{NI}\left(0, \sigma^{2}\right) \quad t=1, \ldots, T, T+1, \ldots, T+H,
$$

where $y_{t}$ is the dependent variable; $z_{t}$ is an exogenous variable; $b$ is an unknown coefficient; $u_{t}$ is an error term, assumed to be independently and normally distributed with a zero mean and a variance $\sigma^{2} ; t$ is the time subscript; and the estimation and forecast periods are $[1, T]$ and $[T+1, T+H]$ respectively, implying $T$ observations for estimation and $H$ observations being forecast.

Several types of model-based forecasts are feasible from (1), each with specific implications for the properties of the corresponding forecasts and forecast errors. For expositional convenience, consider the forecasts based on least squares estimation of the coefficient $b$ in model $\mathrm{M}_{1}$ :

$$
\hat{y}_{t}=\hat{b} z_{t} \quad t=T+1, \ldots, T+H
$$

where $\hat{y}_{t}$ is the forecast of $y_{t}$, and $\hat{b}$ is the least squares estimator of $b$, distributed as $\mathrm{N}\left(b, \sigma^{2}\left(\sum_{t=1}^{T} z_{t}^{2}\right)^{-1}\right)$. To highlight the distinction between the estimation period $[1, T]$ and the forecast period $[T+1, T+H]$, it is helpful to rewrite $(2)$ with a different time subscript:

$$
\hat{y}_{T+h}=\hat{b} z_{T+h} \quad h=1, \ldots, H
$$

where $h$ indexes the forecast horizon.

The forecast $\hat{y}_{T+h}$ in (3) assumes that $z_{T+h}$ is known. That is, in period $T$, the value of $z$ at period $T+h$ (i.e., $h$ periods into the future) is known. While this assumption is sensible for deterministic variables such as an intercept and a linear trend, it is unjustified for many variables, particularly when $h>1$. Section 3.2 explores that issue further, with $z_{t}=y_{t-1}$.

The forecast error $e_{T+h}$ is the difference between the outcome $y_{T+h}$ and its forecast $\hat{y}_{T+h}$ :

$$
\begin{aligned}
e_{T+h} & =y_{T+h}-\hat{y}_{T+h} \\
& =\left(b z_{T+h}+u_{T+h}\right)-\hat{b} z_{T+h} \\
& =u_{T+h}+(b-\hat{b}) z_{T+h} \quad h=1, \ldots, H,
\end{aligned}
$$

where the second line of (4) uses the definitions of $y_{T+h}$ and $\hat{y}_{T+h}$ from (1) and (3) respectively, and the third line re-arranges terms. So, from (4), the forecast error $e_{T+h}$ is comprised of the (unknown) future shock $u_{T+h}$, and $(b-\hat{b}) z_{T+h}$, which is the error in forecasting that arises from estimating rather than knowing the coefficient $b$. These 
are the components $1(\mathrm{a})$ and $1(\mathrm{~b})$ in Table 1, with the two components together representing "what we know that we don't know" about the future outcome $y_{T+h}$.

Many measures exist for summarizing properties of a forecast and its corresponding forecast error, including the bias of the forecast, the variance of the forecast error, and the mean square forecast error (MSFE), which is a common summary measure of a forecast error's properties. The MSFE is defined as the mean of the squared forecast error, e.g., $\mathcal{E}\left(e_{T+h}^{2}\right)$ for the forecast error $e_{T+h}$ in (4). In general, the whole distribution of outcomes is of interest when considering forecast uncertainty. That said, the primary measure of forecast uncertainty in economics is the MSFE, which simplifies to the variance of the forecast error when the forecast is unbiased. The variance and MSFE capture important aspects of forecast uncertainty and so are considered in much of the discussion below. Still, they have substantive limitations as measures of forecast uncertainty; see Clements and Hendry (1993) for a detailed analysis.

For the static model (1), the forecast error (4) has easily derivable properties. In fact, its distribution is normal:

$$
e_{T+h} \sim \mathrm{N}\left(0, \sigma^{2}\left[1+z_{T+h}^{2}\left(\sum_{t=1}^{T} z_{t}^{2}\right)^{-1}\right]\right) \quad h=1, \ldots, H
$$

as follows immediately from normality and independence of $\left\{u_{t}\right\}$, and hence the normality and independence of $u_{T+h}$ and $\hat{b}$. Further results depend on the nature of $z_{t}$. For the simple case where $z_{t}$ is an intercept (i.e., $z_{t}=1$ ), then (5) simplifies:

$$
e_{T+h} \sim \mathrm{N}\left(0, \sigma^{2}[1+(1 / T)]\right) \quad h=1, \ldots, H
$$

Because the forecast $\hat{y}_{T+h}$ in (3) is unbiased for the outcome $y_{T+h}$, the variance of $e_{T+h}$ is also the MSFE.

Figure 1a (the upper left graph in Figure 1) plots the MSFE for $b=0.5$ as a function of the forecast horizon $h$, where the units are in terms of $\sigma^{2}$. Figure 1a contains two calculations of the MSFE. The first is the variance in (6) for a large estimation sample (i.e., $T=\infty$ ) and so is denoted the asymptotic MSFE ("no estimation uncertainty"). From (6), the asymptotic MSFE equals $\sigma^{2}$ for all forecast horizons: hence it is a flat line at unity in the graph. The second MSFE is the variance in (6) for a specific estimation sample (here, $T=5$ ) and so is denoted the finite sample MSFE ("with estimation uncertainty"). ${ }^{2}$ From (6), it numerically equals $1.20 \sigma^{2}$.

In summary, for a static model with only an intercept, the predictable uncertainty as measured by the MSFE is constant across forecast horizons. The MSFE decreases as the estimation sample increases, tending to a positive value (the asymptotic MSFE)

\footnotetext{
${ }^{2}$ While a sample size of $T=5$ seems very small, it may not be so in this context, where only a single parameter is estimated. In unrestricted vector autoregressive models and autoregressive distributed lag models, the number of estimated parameters per equation is commonly $10 \%-30 \%$ of the sample size - similar to the percentage here.
} 

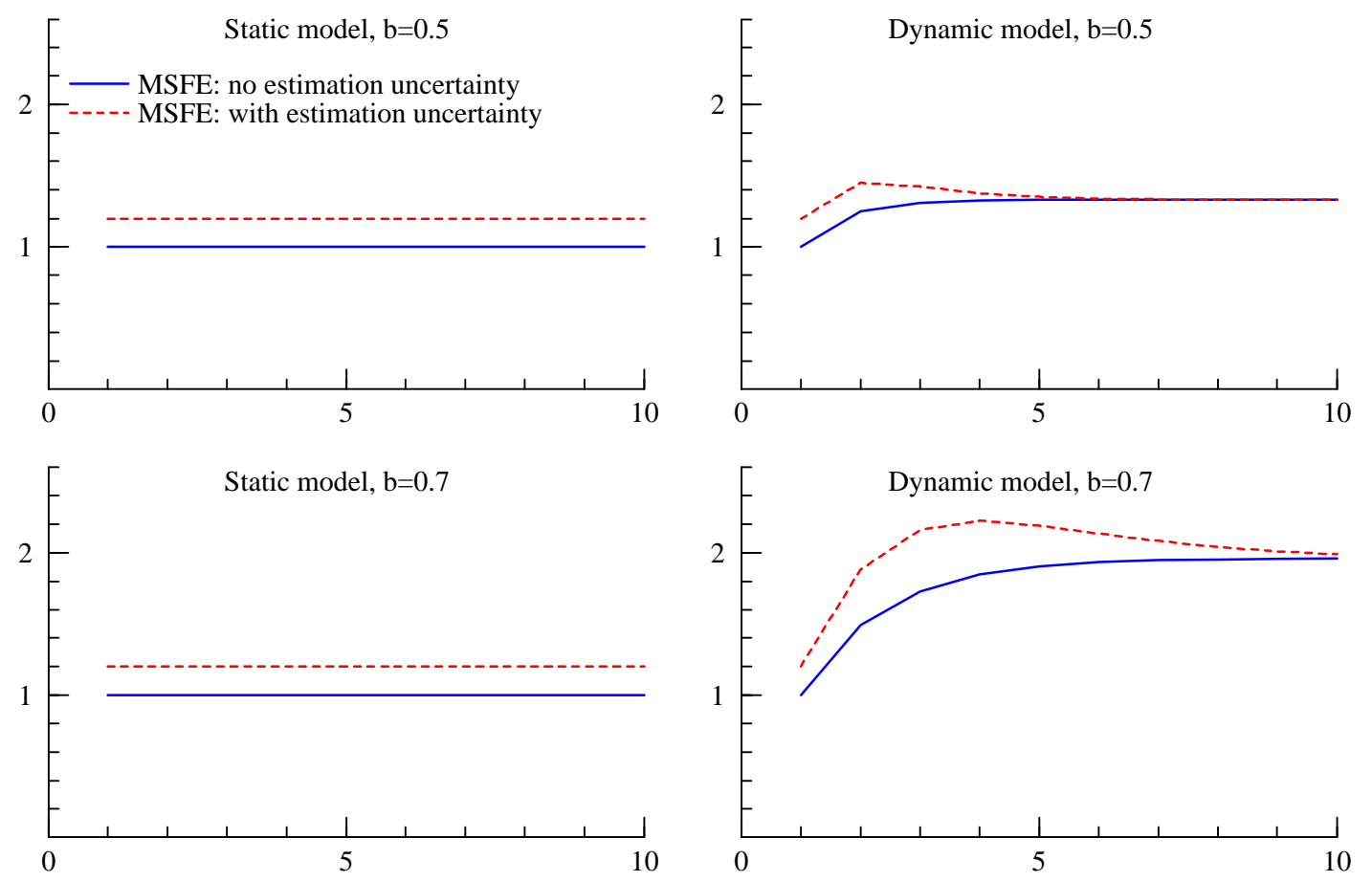

Figure 1: Asymptotic and finite sample mean square forecast errors (MSFEs) for static and dynamic models with coefficient values of $b=0.5$ and $b=0.7$.

for a large estimation sample. For static regression models in general, the asymptotic MSFE is invariant to the forecast horizon; in finite samples, it may depend on both $h$ and $T$ through the component involving estimation uncertainty.

\subsection{A Dynamic Single Equation}

This subsection describes the model, model-based forecasts, and forecast errors for a simple dynamic model - specifically, for a univariate first-order autoregressive model. This model has been extensively studied analytically, numerically, and empirically in the statistical, econometric, and economic literature; see Orcutt and Winokur (1969), Hoque, Magnus, and Pesaran (1988), and Clements and Hendry (1998, Chapter 4) inter alia on the model's forecast properties. This model highlights the effects of dynamics on forecasts, and many of its forecasts' properties generalize readily to systems of dynamic equations with multiple lags; see Section 4.

The first-order autoregressive model (denoted $\left.\mathrm{M}_{2}\right)$ is:

$$
\mathrm{M}_{2}: \quad y_{t}=b y_{t-1}+u_{t} \quad u_{t} \sim \mathrm{NI}\left(0, \sigma^{2}\right) \quad t=1, \ldots, T, T+1, \ldots, T+H,
$$

where $b$ is now the unknown coefficient on the lagged dependent variable $y_{t-1}$. The 
variable $z_{t}$ in model $\mathrm{M}_{1}$ is now $y_{t-1}$, but the properties of the error term $u_{t}$ and the notation are otherwise as in model $\mathrm{M}_{1}$ (Section 3.1). For expositional convenience, it is assumed that $|b|<1$ and that $y_{0} \sim \mathrm{N}\left(0, \sigma^{2} /\left(1-b^{2}\right)\right){ }^{3}$

The forecasts considered are those based on least squares estimation of the coefficient $b$ in model $\mathrm{M}_{2}$ :

$$
\hat{y}_{t}=\hat{b} y_{t-1} \quad t=T+1, \ldots, T+H,
$$

where $\hat{b}$ is the least squares estimator of the coefficient $b$, and is approximately distributed as $N\left(b,\left(1-b^{2}\right) / T\right)$. As with (2), equation (8) may be re-indexed, resulting in the following sequence of one-step ahead forecasts:

$$
\hat{y}_{T+h}=\hat{b} y_{T+h-1} \quad h=1, \ldots, H .
$$

Equation (9) may be written more explicitly as:

$$
\begin{aligned}
\hat{y}_{T+1} & =\hat{b} y_{T} \\
\hat{y}_{T+2} & =\hat{b} y_{T+1} \\
& \vdots \\
\hat{y}_{T+h} & =\hat{b} y_{T+h-1} \\
& \vdots \\
\hat{y}_{T+H-1} & =\hat{b} y_{T+H-2} \\
\hat{y}_{T+H} & =\hat{b} y_{T+H-1} .
\end{aligned}
$$

Equation (10) highlights a complication when forecasting from dynamic models: the one-period ahead forecast $\hat{y}_{T+1}$ is easily constructed because $y_{T}$ is known, but forecasts at longer horizons depend upon future (unknown) outcomes of $y$. The usual approach is to replace those future outcomes by their forecasts. Thus, the forecast $\hat{y}_{T+2}$ becomes $\hat{b} \cdot\left(\hat{b} y_{T}\right)$ and, more generally, the $h$-period ahead forecast $\hat{y}_{T+h}$ is:

$$
\hat{y}_{T+h}=\hat{b}^{h} y_{T} \quad h=1, \ldots, H .
$$

The forecast error $e_{T+h}$ is the difference between the outcome $y_{T+h}$ and its forecast $\hat{y}_{T+h}$, as in the first line of (4), but the analytics differ from (4). To calculate the forecast error, it is helpful first to derive $y_{T+h}$ in terms of the observed $y_{T+i}$ (for $i<h$ ) and the unobserved future shocks $\left\{u_{T+1}, u_{T+2}, \ldots, u_{T+h}\right\}$. By repeated substitution from $(7), y_{T+h}$ is:

$$
\begin{aligned}
y_{T+h} & =b y_{T+h-1}+u_{T+h} \\
& =b \cdot\left(b y_{T+h-2}+u_{T+h-1}\right)+u_{T+h} \\
& \vdots \\
& =b^{h} y_{T}+\sum_{i=0}^{h-1} b^{i} u_{T+h-i} .
\end{aligned}
$$

\footnotetext{
${ }^{3}$ These assumptions imply that $y_{t}$ is integrated of order zero and ergodic. See Banerjee, Dolado, Galbraith, and Hendry (1993) for details.
} 
Thus, the forecast error $e_{T+h}$ is:

$$
\begin{aligned}
e_{T+h} & =y_{T+h}-\hat{y}_{T+h} \\
& =\left(b^{h} y_{T}+\sum_{i=0}^{h-1} b^{i} u_{T+h-i}\right)-\hat{b}^{h} y_{T} \\
& =\sum_{i=0}^{h-1} b^{i} u_{T+h-i}+\left(b^{h}-\hat{b}^{h}\right) y_{T} \quad h=1, \ldots, H .
\end{aligned}
$$

As with the static model, the dynamic model's forecast error depends upon future shocks to the economy and upon estimating rather than knowing the coefficient $b$. The static and dynamic forecast errors do differ in the form of their dependence on those two components, as comparison of (4) and (13) clarifies. First, the forecast error of the static model depends on the future shock in period $T+h$ alone, whereas the dynamic model's forecast error contains a weighted cumulation of shocks from period $T+1$ through period $T+h$. Second, the static model's forecast error depends on the simple difference between the coefficient $b$ and its estimate $\hat{b}$. The dynamic model's forecast error depends upon the difference between powers of $b$ and $\hat{b}$. For the one-period ahead forecast, the dynamic model's forecast error is $u_{T+1}+(b-\hat{b}) y_{T}$, paralleling the static model's forecast error of $u_{T+1}+(b-\hat{b}) z_{T+1}$. At other horizons, forecasts errors from the two types of models differ in detail, even while each has the two components 1(a) and 1(b) from Table 1.

From (13), the properties of the dynamic model's forecast errors depend upon the distribution of $\sum_{i=0}^{h-1} b^{i} u_{T+h-i}$ and $\left(b^{h}-\hat{b}^{h}\right)$, and upon the initial condition for forecasting, i.e., $y_{T}$. While $\sum_{i=0}^{h-1} b^{i} u_{T+h-i}$ is visually the most complex of the three terms, its distribution is the most straightforward, as the future shocks $\left\{u_{T+h-i}\right\}$ are normally distributed and serially independent. The mean of $\sum_{i=0}^{h-1} b^{i} u_{T+h-i}$ is zero, and its variance is the sum of the variances of its components:

$$
\begin{aligned}
\operatorname{var}\left(\sum_{i=0}^{h-1} b^{i} u_{T+h-i}\right) & =\operatorname{var}\left(u_{T+h}\right)+\operatorname{var}\left(b u_{T+h-1}\right)+\cdots+\operatorname{var}\left(b^{h-1} u_{T+1}\right) \\
& =\sigma^{2}+b^{2} \sigma^{2}+\cdots+b^{2(h-1)} \sigma^{2} \\
& =\sigma^{2}\left(\frac{1-b^{2 h}}{1-b^{2}}\right) .
\end{aligned}
$$

The exact distribution of the forecast errors is complicated by the nonlinearity $\hat{b}^{h}$ and by the dependence of $\hat{b}$ on $y_{T}$. However, under one standard set of approximations, $\left(b^{h}-\hat{b}^{h}\right)$ is distributed as $\mathrm{N}\left(0,\left[\left(h b^{h-1}\right)^{2}\left(1-b^{2}\right) / T\right]\right)$, the variable $y_{T}$ has its unconditional distribution $\mathrm{N}\left(0,\left[\sigma^{2} /\left(1-b^{2}\right)\right]\right)$, and the term $\left(b^{h}-\hat{b}^{h}\right)$ and $y_{T}$ are independently distributed. Using these approximations, the forecast error from the dynamic model has a mean of (approximately) zero and a variance (approximately) equal to:

$$
\sigma^{2}\left(\frac{1-b^{2 h}}{1-b^{2}}\right)+\frac{\sigma^{2}\left(h b^{h-1}\right)^{2}}{T},
$$

which is also its (approximate) finite sample MSFE. The first term in (15) is the asymptotic MSFE in (14). The second term captures the complicated dependence of 
$\left(b^{h}-\hat{b}^{h}\right) y_{T}$ on four factors: the forecast horizon $h$, the autoregressive coefficient $b$, the estimation sample size $T$, and the equation error variance $\sigma^{2}$.

Figure 1b plots the asymptotic MSFE in (14) as a function of the forecast horizon $h$. From (14), the asymptotic MSFE equals $\sigma^{2}$ for $h=1, \sigma^{2}+b^{2} \sigma^{2}$ for $h=2$, $\sigma^{2}+b^{2} \sigma^{2}+b^{4} \sigma^{2}$ for $h=3$, and so on, tending to $\sigma^{2} /\left(1-b^{2}\right)$ for large $h$. In Figure $1 \mathrm{~b}$, $b=0.5$, so these values are $\sigma^{2}, 1.25 \sigma^{2}, 1.31 \sigma^{2}$, and so on, tending to $1.33 \sigma^{2}$. The (asymptotic) predictable uncertainty increases as the forecast horizon increases; and that predictable uncertainty is bounded over all forecast horizons.

Figure $1 \mathrm{~b}$ also plots the (approximate) finite sample MSFE in (15). This MSFE is larger than (or at least as large as) the asymptotic MSFE: estimation uncertainty adds to the inherent uncertainty from the future shocks, as for the static model. The finite sample MSFE is also bounded over all forecast horizons. As Figure 1b demonstrates, the finite sample MSFE need not increase monotonically in the forecast horizon: as $h$ increases, the finite sample MSFE may decrease as well as increase. The explanation for this non-monotonicity turns on the term $\sigma^{2}\left(h b^{h-1}\right)^{2} / T$ in (15). That term has a

complicated dependence on the forecast horizon $h$, but in any case is always positive for nonzero $b$ and tends to zero as $h$ becomes large. For economic and policy analysis, non-monotonicity in the MSFE can be worrying: the forecast horizons of greatest economic or policy interest may have the greatest (predictable) uncertainty.

To summarize, for a dynamic first-order autoregressive model, the predictable uncertainty as measured by the MSFE tends to increase in the forecast horizon, although estimation uncertainty may induce non-monotonicity. As with the MSFE from the static model, the dynamic model's MSFE decreases as the estimation sample increases, tending to a positive value (the asymptotic MSFE) for a large estimation sample. The MSFEs from dynamic and static models differ most notably through their dependence (or lack thereof) on the forecast horizon. The following subsection, along with Section 4, examines this issue in greater detail.

\subsection{A Comparison}

This subsection compares the predictive uncertainty from the static and dynamic models in the two previous subsections, focusing on the roles of coefficient estimation and the forecast horizon. To highlight the latter, two empirical models of U.K. net national income are examined.

Figures 1a and 1b plotted the MSFEs for the static and dynamic models, evaluated at a specific value of the regression parameter $b: b=0.5$. Figures $1 c$ and $1 \mathrm{~d}$ plot the comparable graphs for a different value of $b: b=0.7$. For the static model (Figure 1c), both the asymptotic and finite sample MSFEs remain unchanged. In fact, the MSFEs for the static model are invariant to $b$, as (5) and (6) imply. For the dynamic model (Figure 1d), both the asymptotic and finite sample MSFEs have altered. Each increases more rapidly at small forecast horizons; and they tend to a 
larger MSFE for large $h$ : to $1.96 \sigma^{2}$, rather than to $1.33 \sigma^{2}$. For multi-period ahead forecasts at a given horizon $h$, both the asymptotic and finite sample MSFEs increase in the coefficient $b$, as follows from the sum in the second line of (14) and from the second term in (15).

Thus, the static nature of the static model over the estimation (in-sample) period carries through to the (predictable) properties of its forecasts and forecast errors. Time dependence characterizes the nature of the dynamic model, both in sample and over the forecast period. These results show that characteristics of forecasts are model dependent. Somewhat surprisingly, characteristics of forecasts are relatively data independent.

To illustrate these results, the remainder of this subsection considers two models - one static and the other dynamic - that generalize or expand upon the models in Sections 3.1 and 3.2. The first is a regression model with an intercept and linear trend, and the second is a random walk model with drift. Both have played important roles in statistical and economic modelling; see Box and Jenkins (1970), Hendry, Pagan, and Sargan (1984), Hendry (1995, Chapter 7), and Clements and Hendry (2000) inter alia. For ease of exposition, uncertainty from coefficient estimation is ignored.

The first model is a static model with both an intercept and a linear trend:

$$
\mathrm{M}_{1}^{*}: \quad y_{t}=b_{1}+b_{2} t+u_{t} \quad u_{t} \sim \mathrm{NI}\left(0, \sigma^{2}\right) \quad t=1, \ldots, T, T+1, \ldots, T+H,
$$

where $b_{1}$ is the intercept and $b_{2}$ is the coefficient on the trend $t$. The forecasts are:

$$
\hat{y}_{T+h}=b_{1}+b_{2} \cdot(T+h) \quad h=1, \ldots, H,
$$

so the forecast errors are:

$$
e_{T+h}=u_{T+h} \quad h=1, \ldots, H
$$

and the MSFE is:

$$
\operatorname{var}\left(e_{T+h}\right)=\sigma^{2} \quad h=1, \ldots, H .
$$

As in the simpler static model with only an intercept, the (asymptotic) MSFE does not depend upon the forecast horizon $h$.

The MSFE in (19) is the "predicted" MSFE, in that $\sigma^{2}$ is the value of the MSFE, assuming that model $\mathrm{M}_{1}^{*}$ in (16) is the DGP. With some distributional assumptions about $u$, the predicted MSFE can provide the basis for calculating confidence intervals for the forecasts in (17), as is common in many econometrics software packages. In general, the predicted MSFE differs from the actual MSFE, as when the assumed model is not the DGP; see Clements and Hendry (2000).

To illustrate the properties of the predicted MSFE empirically, consider modelling real net national income in the United Kingdom over 1970-1993 and forecasting it through 2010. The data are from Ericsson, Hendry, and Prestwich (1998), and the 

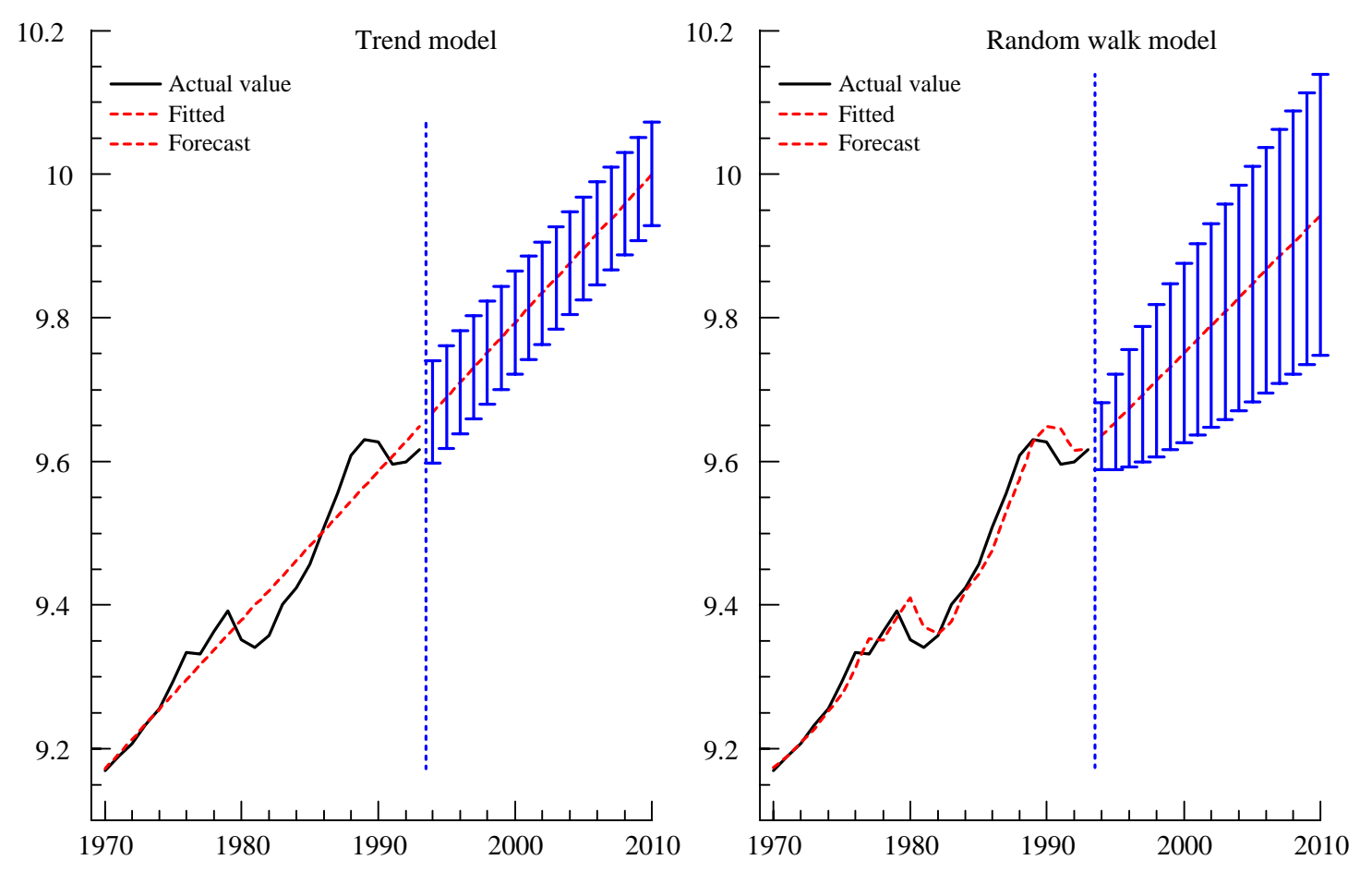

Figure 2: Actual, fitted, and forecast values from the trend and random walk models of annual real net national income for the United Kingdom (in logs), with $95 \%$ confidence intervals for the forecasts.

model is (16), where $y$ is the logarithm of real net national income. Figure 2a shows the results from estimating this model and forecasting from it. The left half of Figure 2a plots actual income and the fitted values of income from the estimated model over 1970-1993. The right half of Figure 2a plots the model's forecasts through 2010. The vertical bars around those forecasts represent the anticipated $95 \%$ confidence intervals for income, which are roughly $\pm 2 \hat{\sigma}$ (namely, plus-or-minus twice the estimated equation standard error). While this model for income does include a trend, that trend is deterministic, implying that its future values are known, as well as its current and past values. So, in essence, this model is static, and the anticipated forecast uncertainty is constant across different forecast horizons.

The second model is a random walk with an intercept:

$$
\mathrm{M}_{2}^{*}: \quad y_{t}=a+y_{t-1}+u_{t} \quad u_{t} \sim \mathrm{NI}\left(0, \sigma^{2}\right) \quad t=1, \ldots, T, T+1, \ldots, T+H,
$$

where $a$ is the intercept, and the coefficient on the lagged dependent variable is constrained to equal unity. 
Following (11), (13), and the first two lines of (14), the $h$-step ahead forecasts are:

$$
\hat{y}_{T+h}=a h+y_{T} \quad h=1, \ldots, H
$$

the forecast errors are:

$$
e_{T+h}=\sum_{i=0}^{h-1} u_{T+h-i} \quad h=1, \ldots, H
$$

and the MSFE is:

$$
\operatorname{var}\left(e_{T+h}\right)=h \sigma^{2} \quad h=1, \ldots, H .
$$

The predicted MSFE increases in the forecast horizon $h$, and in fact increases linearly in $h$, and without bound. The predicted MSFE in (23) assumes that model $\mathrm{M}_{2}^{*}$ in (20) is the DGP.

Figure $2 \mathrm{~b}$ plots the actual, fitted, and forecast values from this random walk model of income, using the same sample periods for estimation and forecasting as with the static model in Figure 2a. The confidence intervals for the random walk forecasts in Figure $2 \mathrm{~b}$ increase very substantially as the forecast horizon itself increases, contrasting with confidence intervals of fixed width in Figure 2a.

Figures $2 \mathrm{a}$ and $2 \mathrm{~b}$ portray two very different patterns for the anticipated (or predicted) forecast uncertainty, and their comparison illustrates how model choice can affect those patterns. Exactly the same series is being modelled and forecast in Figures $2 \mathrm{a}$ and 2b: only the models themselves differ. More generally, static models often imply predicted forecast uncertainty that is time invariant or nearly so, whereas dynamic models generally imply time-dependent predicted forecast uncertainty, often increasing in the forecast horizon.

Models $\mathrm{M}_{1}$ and $\mathrm{M}_{2}$ present static and dynamic relationships as black and white, but in practice a whole spectrum of models exists with both static and dynamic aspects. The simplest example is:

$$
\mathrm{M}_{3}: \quad y_{t}=b_{1} y_{t-1}+b_{2} z_{t}+u_{t}
$$

where $b_{1}$ and $b_{2}$ are the coefficients of lagged $y$ and current $z$. Model $\mathrm{M}_{3}$ includes models $\mathrm{M}_{1}$ and $\mathrm{M}_{2}$ as special cases with $b_{1}=0$ and $b_{2}=0$ respectively. When $b_{1}$ and $b_{2}$ are both nonzero, model $\mathrm{M}_{3}$ has both dynamic and static features. Analytical examination of model $\mathrm{M}_{3}$ 's predictable forecast uncertainty is feasible but reveals relatively little beyond what has been seen from models $M_{1}$ and $M_{2}$ separately.

This section has considered models and forecasts of individual variables only, and not of sets of variables. Yet, economics typically is about relationships between variables, where those variables may interact with each other, either contemporaneously, or at a lag, or both. The next section thus turns to system forecasts and their associated predictable uncertainty. In the language of Table 2 , the issues are the dimension and completeness of the relationship of interest and of the forecast. 


\section{Predictable Uncertainty for Systems}

In Section 3.1 above, future values of the exogenous variable $z$ were assumed to be known when forecasting $y$. This assumption is sensible for deterministic variables such as an intercept or a linear trend. However, for most economic variables, the variables themselves would need to be forecast when forecasting $y$. Doing so leads directly to forecasting from systems of equations. This section first considers a simple but historically important two-variable system (Section 4.1) and then turns to a vector autoregressive model (or VAR, Section 4.2), which is the basis for much empirical forecasting in economics. As in Section 3, the focus is on the inherent predictable uncertainty. The predictable uncertainty from estimation is calculable, both analytically and numerically, but it provides less direct insight due to the increased complexity of the models.

\subsection{A Simple Dynamic System}

The static model in (1) expresses $y_{t}$ in terms of $z_{t}$ and a disturbance $u_{t}$. This subsection augments that model with an equation for $z_{t}$ itself, so that forecasts of $y$ can be constructed from forecasts of $z$ when the future values of $z$ are unknown.

Many possible models exist for $z_{t}$. One plausible determinant of $z_{t}$ is $y_{t-1}$ : that is, $y$ Granger-causes $z$. See Granger (1969) for the development of the concept of Granger causality, and Hendry and Mizon (1999) for reasons why Granger causality is so prevalent in economic data.

Thus, it is of interest to consider model $\mathrm{M}_{4}$ :

$$
\mathrm{M}_{4}:\left\{\begin{array}{l}
y_{t}=b z_{t}+u_{t} \\
z_{t}=c y_{t-1}+v_{t}
\end{array} \quad\left[\begin{array}{l}
u_{t} \\
v_{t}
\end{array}\right] \sim \mathrm{NI}\left(\left[\begin{array}{l}
0 \\
0
\end{array}\right],\left[\begin{array}{ll}
\sigma^{2} & 0 \\
0 & \omega^{2}
\end{array}\right]\right),\right.
$$

where $c$ is the coefficient on lagged $y$ in the equation for $z$; and $v_{t}$ is the disturbance in that equation. The two disturbances $\left(u_{t}: v_{t}\right)$ are assumed to be normally and independently distributed, having mean zero and (respectively) variances $\sigma^{2}$ and $\omega^{2}$. The covariance between $u_{t}$ and $v_{t}$ is zero: that is, the equation for $y$ is a conditional model of $y_{t}$ given $z_{t}$, and $b$ is the coefficient implied by that conditioning; see Ericsson (1992a) for an introductory exposition on conditioning and (relatedly) exogeneity. These assumptions about normality and conditioning are convenient, but are not central to the properties of the forecasts.

In economics, (25) is known as a cobweb model, which may characterize a market with lags in the production process, as of agricultural commodities. In the cobweb model, $y$ and $z$ are interpreted as the logs of price and quantity, respectively. Denoting those logs as $p$ and $q$, (25) becomes:

$$
\mathrm{M}_{4}^{*}:\left\{\begin{array}{l}
p_{t}=b q_{t}+u_{t} \\
q_{t}=c p_{t-1}+v_{t}
\end{array} \quad\left[\begin{array}{l}
u_{t} \\
v_{t}
\end{array}\right] \sim \mathrm{NI}\left(\left[\begin{array}{l}
0 \\
0
\end{array}\right],\left[\begin{array}{ll}
\sigma^{2} & 0 \\
0 & \omega^{2}
\end{array}\right]\right) .\right.
$$


The cobweb model (26) has the following interpretation. The equation for $p_{t}$ is derived from a demand equation: the price $p_{t}$ clears the market for a given quantity $q_{t}$ supplied. The value $1 / b$ is the price elasticity of demand. The equation for $q_{t}$ is a supply equation, capturing (for instance) how much farmers decide to produce this year $\left(q_{t}\right)$, depending upon the price that they were able to obtain in the previous year $\left(p_{t-1}\right)$. The value $c$ is the price elasticity of supply. See Tinbergen (1931) and Suits (1955) for pivotal contributions on the cobweb model, and Henderson and Quandt (1971, pp. 142-145) for an exposition.

Forecasting from the cobweb model generalizes on the mechanism for forecasting from the first-order autoregressive model in Section 3.2, so we revert to the $(y: z)$ notation in (25). Ignoring estimation, the forecasts derive from (25) without the disturbances, whose future values are by definition unknown:

$$
\left\{\begin{array}{l}
\hat{y}_{T+h}=b z_{T+h} \\
\hat{z}_{T+h}=c y_{T+h-1}
\end{array} \quad h=1, \ldots, H .\right.
$$

Equation (27) may be written more explicitly as:

$$
\begin{aligned}
{\left[\begin{array}{l}
\hat{y}_{T+1} \\
\hat{z}_{T+1}
\end{array}\right] } & =\left[\begin{array}{l}
b z_{T+1} \\
c y_{T}
\end{array}\right] \\
{\left[\begin{array}{l}
\hat{y}_{T+2} \\
\hat{z}_{T+2}
\end{array}\right] } & =\left[\begin{array}{l}
b z_{T+2} \\
c y_{T+1}
\end{array}\right] \\
{\left[\begin{array}{l}
\hat{y}_{T+h} \\
\hat{z}_{T+h}
\end{array}\right] } & =\left[\begin{array}{l}
b z_{T+h} \\
c y_{T+h-1}
\end{array}\right] \\
{\left[\begin{array}{l}
\hat{y}_{T+H-1} \\
\hat{z}_{T+H-1}
\end{array}\right] } & =\left[\begin{array}{l}
b z_{T+H-1} \\
c y_{T+H-2}
\end{array}\right] \\
{\left[\begin{array}{l}
\hat{y}_{T+H} \\
\hat{z}_{T+H}
\end{array}\right] } & =\left[\begin{array}{l}
b z_{T+H} \\
c y_{T+H-1}
\end{array}\right] .
\end{aligned}
$$

Formally, (28) parallels (10), with the former delineating pairs of forecasts at a given horizon $h$, rather than a single forecast at each horizon. All variables except $y_{T}$ on the right-hand side of (28) are dated in the future and hence unknown, so an implementable forecasting algorithm is still required.

Consider generating the forecasts for $y$ first. Substituting the second equation of (27) into the first obtains:

$$
\hat{y}_{T+h}=b c y_{T+h-1} \quad h=1, \ldots, H,
$$

which is identical to (9), except that the autoregressive coefficient is $b c$, rather than $b$ itself. All $H$ forecasts for $y$ can be generated from (29), employing the approach in 
Section 3.2. The $H$ forecasts for $z$ follow immediately from the second line in (27):

$$
\hat{z}_{T+h}=c y_{T+h-1} \quad h=1, \ldots, H
$$

using $c y_{T}$ as the forecast for $z_{T+1}$, and $c \hat{y}_{T+h-1}$ (i.e., with a forecast of $y$ ) as the forecast for $z_{T+h}$ for $h>1$.

For (25), the forecasts for $y$ and $z$ were conveniently solved for $y$ in terms of its own lag, and then for $z$ in terms of lagged $y$. Expositionally, this is clearly convenient. However, for more complicated models, a generic approach is usually taken. Specifically, the one-period ahead $(h=1)$ forecasts for both $y$ and $z$ are derived, as from the first block of rows in (28). The two-period ahead $(h=2)$ forecasts are calculated, using the one-period ahead forecasts. The remaining forecasts of $y$ and $z$ are then solved in this stepwise fashion, period by period.

\subsection{A Generalized Dynamic System}

The approach to forecasting in Section 4.1 suggests a generic formulation for forecasting from dynamic systems. The current subsection discusses that formulation and summarizes the properties of predictable uncertainty from such systems.

In Section 4.1, the model $\mathrm{M}_{4}$ in (25) describes $y$ as a function of contemporaneously dated $z$. By contrast, the forecasting equation for $y$, in (29), solves for $y$ in terms of its lags by substitution with the forecasting equation for $z$. A similar substitution can be applied to the model for $y$, i.e., to the first line of (25). Substituting the second line of (25) into the first obtains:

$$
\begin{aligned}
y_{t} & =b c y_{t-1}+\left(u_{t}+b v_{t}\right) \\
& =b^{*} y_{t-1}+u_{t}^{*}
\end{aligned}
$$

where $b^{*}=b c, u_{t}^{*}=u_{t}+b v_{t}$, and $b^{*}$ and $u_{t}^{*}$ are the reduced form coefficient and disturbance of the equation for $y$. Combining (31) with the equation for $z$ in (25) produces a system for $\left(y_{t}: z_{t}\right)$ that depends on only their lags and a pair of disturbances:

$$
\mathrm{M}_{4}^{\mathrm{rf}}:\left\{\begin{array}{l}
y_{t}=b^{*} y_{t-1}+u_{t}^{*} \\
z_{t}=c y_{t-1}+v_{t}
\end{array} \quad\left[\begin{array}{l}
u_{t}^{*} \\
v_{t}
\end{array}\right] \sim \mathrm{NI}\left(\left[\begin{array}{l}
0 \\
0
\end{array}\right],\left[\begin{array}{ll}
\sigma^{2}+b^{2} \omega^{2} & b \omega^{2} \\
b \omega^{2} & \omega^{2}
\end{array}\right]\right),\right.
$$

where the "rf" in $\mathrm{M}_{4}^{\mathrm{rf}}$ indicates that the model is a reduced form. This model can be rewritten in matrix form:

$$
\left[\begin{array}{l}
y_{t} \\
z_{t}
\end{array}\right]=\left[\begin{array}{ll}
b^{*} & 0 \\
c & 0
\end{array}\right]\left[\begin{array}{l}
y_{t-1} \\
z_{t-1}
\end{array}\right]+\left[\begin{array}{l}
u_{t}^{*} \\
v_{t}
\end{array}\right] \quad\left[\begin{array}{l}
u_{t}^{*} \\
v_{t}
\end{array}\right] \sim \mathrm{NI}\left(\left[\begin{array}{l}
0 \\
0
\end{array}\right],\left[\begin{array}{ll}
\sigma^{2}+b^{2} \omega^{2} & b \omega^{2} \\
b \omega^{2} & \omega^{2}
\end{array}\right]\right)
$$

Equation (33) can be written symbolically in the form:

$$
\mathrm{M}_{5}: \mathbf{x}_{t}=\mathbf{B} \mathbf{x}_{t-1}+\mathbf{w}_{t} \quad \mathbf{w}_{t} \sim \mathrm{NI}(\mathbf{0}, \boldsymbol{\Omega})
$$


where bold characters denote vectors or matrices, as required:

$$
\begin{aligned}
\mathbf{x}_{t} & =\left[\begin{array}{l}
y_{t} \\
z_{t}
\end{array}\right], \\
\mathbf{B} & =\left[\begin{array}{ll}
b^{*} & 0 \\
c & 0
\end{array}\right], \\
\mathbf{w}_{t} & =\left[\begin{array}{l}
u_{t}^{*} \\
v_{t}
\end{array}\right], \text { and } \\
\boldsymbol{\Omega} & =\left[\begin{array}{ll}
\sigma^{2}+b^{2} \omega^{2} & b \omega^{2} \\
b \omega^{2} & \omega^{2}
\end{array}\right] .
\end{aligned}
$$

Equation (34) is the system analog of the first-order univariate autoregressive model in equation (7), and the algebra for constructing forecasts from (7) generalizes directly to constructing forecasts from (34); see Clements and Hendry (1998).

Equation (34) provides a remarkably general framework for forecasting, hence its designation as a separate model - model $M_{5}$. The elements in the feedback matrix B may take a wide range of values, and the covariance matrix $\Omega$ need be only symmetric, positive semi-definite. Equation (34) includes models with multiple lags, as those lags may be "stacked" in $\mathbf{x}_{t}$, with a given lag of a variable (in $\mathbf{x}_{t}$ ) equal to (and defined as) the first lag of that variable with one shorter lag (i.e., in $\mathbf{x}_{t-1}$ ) plus a disturbance that is identically zero. Equation (34) also may include an intercept (i.e., a variable equal to just its own lag, and with a starting value of unity) and a linear trend (i.e., a variable equal to its own lag plus an intercept). More generally, (34) may include exogenous variables whose future values are known through similar deterministic relations. Alternatively, (34) may be viewed as a special case of a dynamic system with exogenous variables, when those exogenous variables are restricted to have coefficients of zero. These two complementary views reflect the two generic systems for forecasting examined in the literature; see Baillie (1979) and Schmidt (1974), who derive and discuss the properties of forecast errors from these two systems. Clements and Hendry $(1998,1999)$ review properties of forecasts from systems and provide extensions; Watson (1994) summarizes general properties of VARs.

Several features characterize the predictable uncertainty associated with forecasts using the univariate first-order autoregressive model (7). Those features also characterize the predictable uncertainty associated with forecasts using the vector autoregressive model (34). Specifically, the MSFE depends upon the forecast horizon $h$ and the feedback matrix B. The asymptotic MSFE increases in $h$, and the finite sample MSFE may decrease in $h$.

Some complications are specific to systems. For instance, linear transformations of $\mathbf{x}$ may change the associated predictable uncertainty. Forecast errors for $y$ and $z$ in (25) may each have a high degree of predictable uncertainty, yet those for $y-z$ 
may have little (predictable) uncertainty, as when $y$ and $z$ are strongly cointegrated, with cointegrating vector $(+1:-1)$. Conversely, $y$ and $z$ may be highly predictable, whereas $y-z$ may be very uncertain, as with the trade balance, which is constructed from the difference between exports and imports. See Campos (1992) and Clements and Hendry (1998) for extensive discussions on transformations of forecasts, and some applications.

\section{Uses of Calculated Predictable Uncertainty}

Calculations of predictable uncertainty serve important roles, both statistically and economically. This section illustrates these roles through a few examples, including forecast confidence intervals, tests of constancy, and fan charts in policy.

Statistically, calculations of predictable uncertainty are the basis for forecast confidence intervals, and for tests of a model's constancy over time. Prior to the realization of outcomes, confidence intervals provide an assessment of "expected" or predicted forecast uncertainty, helping to qualify the forecasts themselves and to give a picture of the expected range of likely outcomes. That is, information about forecast uncertainty is important in addition to the forecast itself.

In Section 3.3, the confidence intervals in Figure 2 for both the trend model and the random walk model were obtained from formulas for the associated predictable uncertainty. Even in more complicated models, predictable uncertainty still may be computed, as in Marquez and Ericsson (1993), who examine the one-period and multiple-period ahead forecasts from various nonlinear, multiple-equation models of the U.S. trade balance. Figure 3 plots the forecasts, outcomes, and $95 \%$ forecast confidence intervals for two of their models, both one quarter ahead and multiple quarters ahead. ${ }^{4}$ The forecast period is 1985Q1-1987Q4. For the first, near-static model, the width of the confidence interval varies only slightly with the forecast horizon. For the second, highly dynamic model, the confidence interval depends strongly upon the forecast horizon, with much greater forecast uncertainty at longer horizons. The multi-period ahead confidence intervals for these two models parallel in character the confidence intervals in Figure 2 for the trend and random walk models. In particular, the fan shape of the confidence intervals for the second trade model and the random walk model suggests the very dynamic nature of the variables being forecast and of the models being used to forecast it.

Predictable forecast uncertainty also can help evaluate the models from which the forecasts were generated. For instance, if the forecast errors lie well outside the range that was anticipated, that indicates specification problems with the model. Predictable forecast uncertainty thus permits assessing how important unpredictable forecast uncertainty is in contributing to the realized forecast error — that is, in

\footnotetext{
${ }^{4}$ In the notation of Marquez and Ericsson (1993), the models are Models M1 and M5.
} 

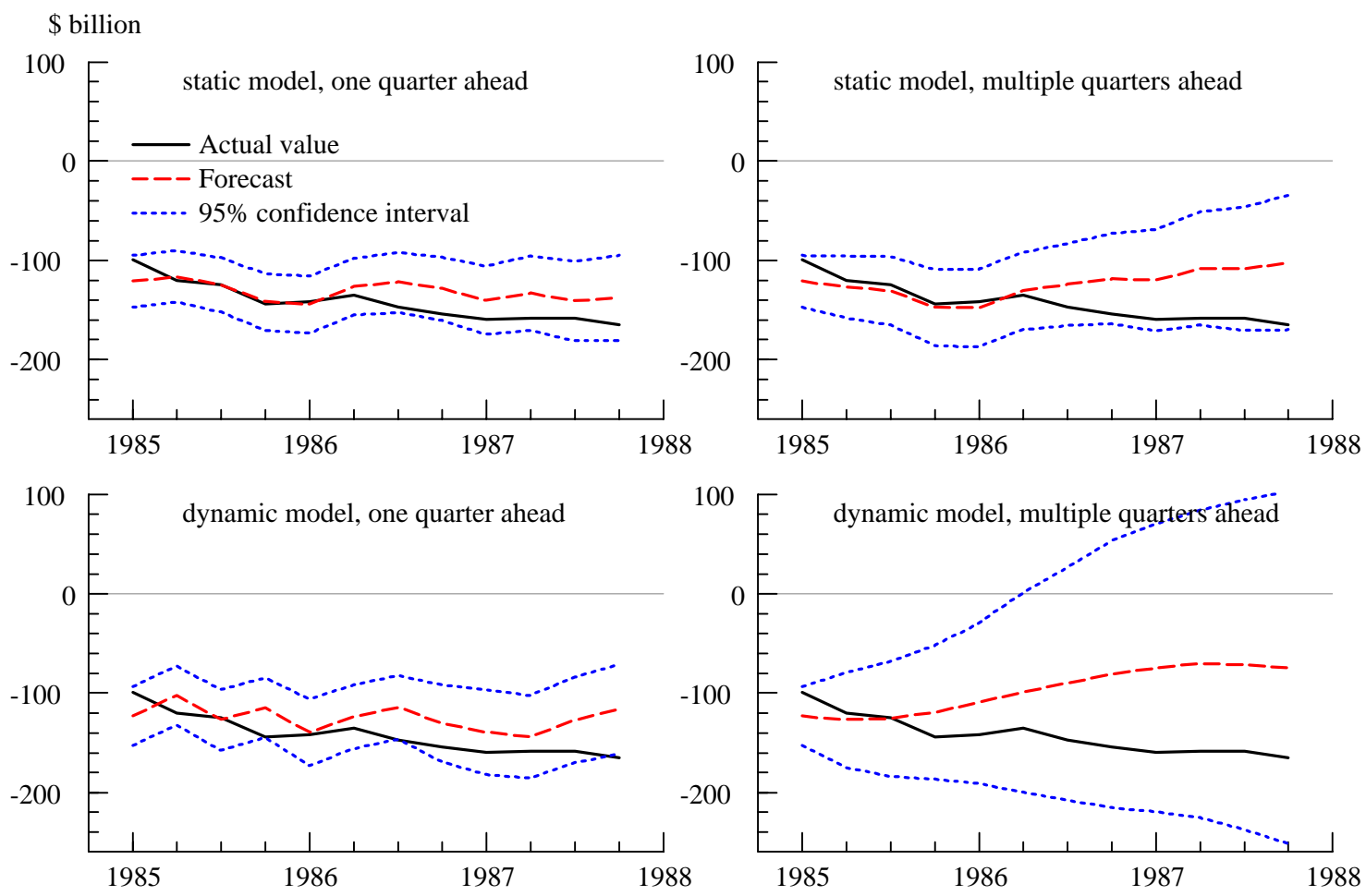

Figure 3: Actual and forecast values from two models of the real U.S. trade balance, both one quarter and multiple quarters ahead, with $95 \%$ confidence intervals for the forecasts.

determining the empirical role of Items $2(\mathrm{a})-2(\mathrm{c})$ in the forecast error taxonomy of Table 1. Specifically, Items 2(a)-2(c) reflect discrepancies between the model used for forecasting and the actual behavior of the economy, with Item 2(a) typically being primarily responsible for forecast failure in econometric models.

Formal statistical analysis of model-based forecasts in light of predictable forecast uncertainty - through tests of parameter constancy - has been central to evaluating and improving empirical economic models. Chow (1960) provides the initial development of these evaluation techniques, building on Fisher (1922), with Hansen (1992a, 1992b) and Andrews (1993) constructing constancy tests with an unknown breakpoint. Goldfeld (1973, 1976), Judd and Scadding (1982), and Baba, Hendry, and Starr (1992) examine constancy tests in their central role in modelling the demand for money. At a more general level, constancy tests are key to evaluating the Lucas (1976) critique, with constancy tests providing the primary empirical basis for refuting the Lucas critique; see Hendry (1988), Engle and Hendry (1993), Ericsson and Irons (1995), and Hendry (2000). Predictable forecast uncertainty is also central to calculating forecast encompassing test statistics; see Chong and Hendry (1986), 
Granger (1989), Ericsson (1992b), Ericsson and Marquez (1993), and West (1996).

Even as a statistical tool, measures of predictable forecast uncertainty have immediate economic implications. For instance, if the forecast uncertainty for a certain variable is viewed as being considerable, insurance might be desirable as a mechanism for protecting against untoward outcomes; and different types of insurance might be available. Also, forecast uncertainty is inherent to many economic activities, such as business investment, with the possibility of large successes often being an attraction of such investment. Forecast uncertainty is ubiquitous in economics, and many consequences follow from the presence and extent of that uncertainty.

Predictable forecast uncertainty has recently taken a visible role in the economic policy arena - in the Bank of England's "fan charts". For the last few years, the Bank of England has published its assessment of (predictable) forecast uncertainty through fan charts for its forecasts of both inflation and GDP growth; see the Bank of England (2000, Overview and Section 6). Figure 4 reproduces the Bank's fan chart (their Chart 6.2, on p. 64) for its November 2000 forecast of inflation. The Bank describes this graph as follows.

The fan chart depicting the probability distribution for inflation is rather like a contour map. At any given point during the forecast period, the depth of shading represents the height of the probability density function over a range of outcomes for inflation. The darkest band includes the central (single most likely) projection and covers $10 \%$ of the probability. Each successive pair of bands is drawn to cover a further $10 \%$ of the probability, until $90 \%$ of the probability distribution is covered. The bands widen as the time horizon is extended, indicating increasing uncertainty about outcomes. Bank of England (2000, Chart 6.2, p. 64)

This fan chart summarizes the Bank's predicted or anticipated probability distribution of inflation outcomes. The Bank of England (2000, Chart 6.4, p. 66) also publishes the density function corresponding to that distribution, which appears in Figure 5. From Figure 5, the Bank's 90\% confidence interval for annual inflation in the twelve months 2002Q1-2002Q4 is from about $1.2 \%$ to $3.9 \%$, as indicated by the shaded area in the graph. Outcomes for inflation could occur outside that range, but the probability of those outcomes is believed to be relatively small.

Improvements to the Bank's fan charts may be feasible; see Wallis (1999a). In the future, economists also could examine whether the Bank of England's published confidence bands reflect what happened in the data, or if those bands were too narrow or too wide. Such an analysis could benefit the construction of future monetary policy. Thus, the statistical and economic aspects of predictable uncertainty are often closely intertwined. 


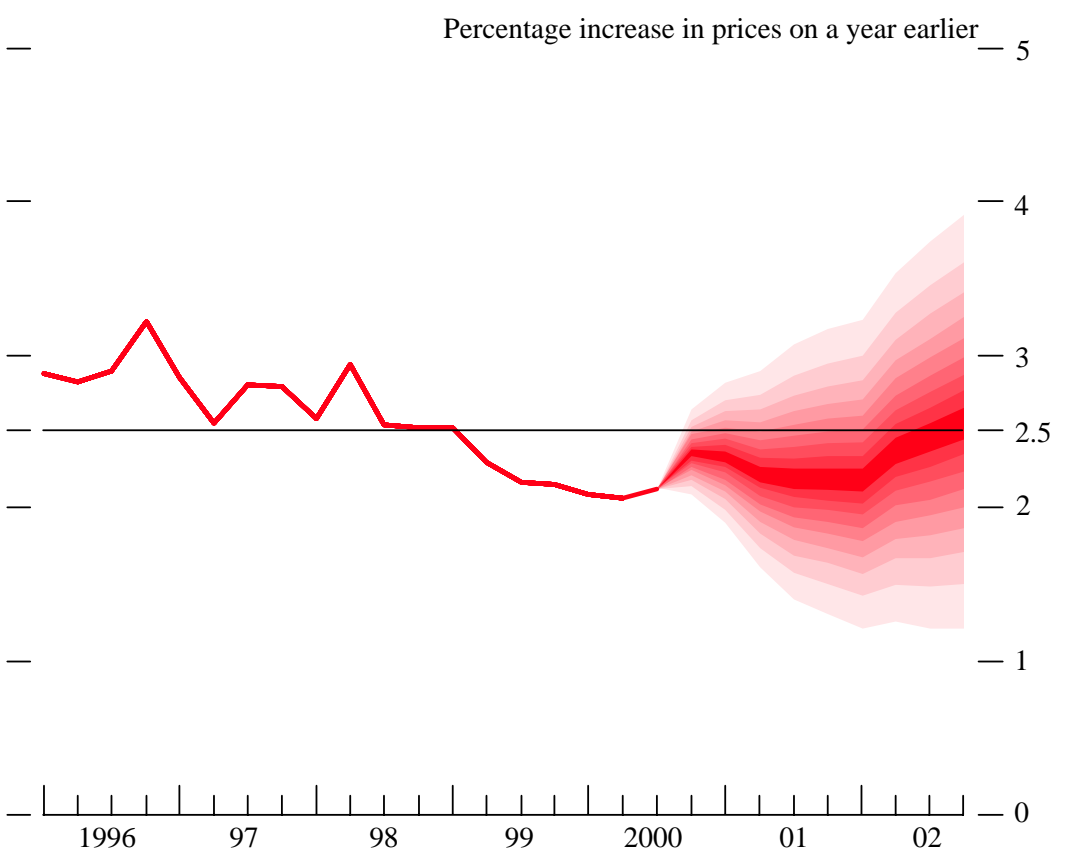

Figure 4: The Bank of England's November 2000 fan chart for projections of RPIX inflation.

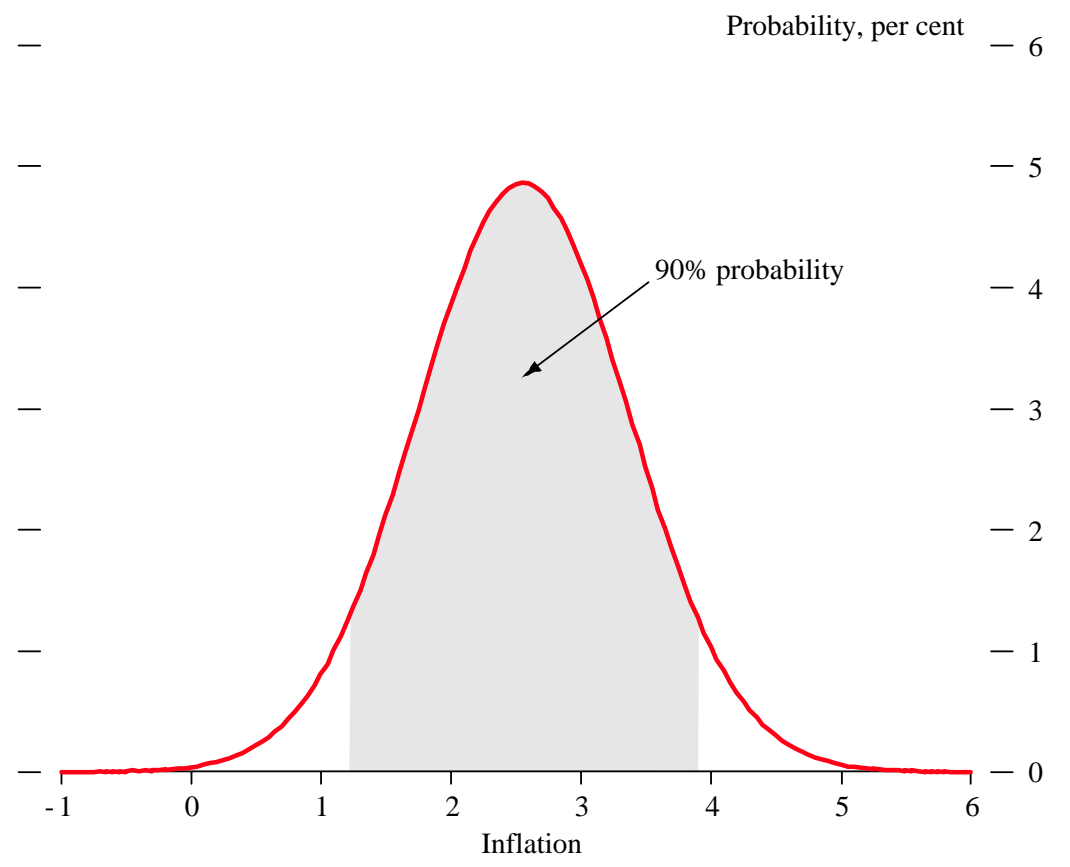

Figure 5: The November 2000 projection by the Bank of England for the probability density of RPIX inflation in the year to 2002Q4. 


\section{Conclusions}

Forecast uncertainty reflects the dispersion of possible outcomes relative to the forecast being made. Forecast uncertainty arises both from "what we don't know that we don't know" and from "what we know that we don't know". In econometric models, forecast uncertainty from the latter - predictable forecast uncertainty can be calculated numerically. Forecast uncertainty also depends upon the variable being forecast, the type of model used for forecasting, the economic process actually determining the variable being forecast, and the forecast horizon.

Calculation of predictable forecast uncertainty has numerous uses in economic practice. First, prior to the realization of outcomes, it helps in qualifying the forecasts themselves and in giving a picture of the expected range of likely outcomes. Information about forecast uncertainty is important in addition to the forecast itself, as with the Bank of England's fan charts. Measures of forecast uncertainty also provide economists with a tool for assessing the importance of unmodelled features of the economy, both directly through the calculated forecast uncertainty, and indirectly through comparison of that calculated uncertainty with the realized distribution of forecast errors. 


\section{References}

Andrews, D. W. K. (1993) "Tests for Parameter Instability and Structural Change With Unknown Change Point", Econometrica, 61, 4, 821-856.

Baba, Y., D. F. Hendry, and R. M. Starr (1992) "The Demand for M1 in the U.S.A., 1960-1988", Review of Economic Studies, 59, 1, 25-61.

Baillie, R. T. (1979) "Asymptotic Prediction Mean Squared Error for Vector Autoregressive Models", Biometrika, 66, 3, 675-678.

Banerjee, A., J. J. Dolado, J. W. Galbraith, and D. F. Hendry (1993) Co-integration, Error Correction, and the Econometric Analysis of Non-stationary Data, Oxford University Press, Oxford.

Bank of England (2000) Inflation Report: November 2000, Bank of England, London.

Box, G. E. P., and G. M. Jenkins (1970) Time Series Analysis: Forecasting and Control, Holden-Day, San Francisco.

Campos, J. (1992) "Confidence Intervals for Linear Combinations of Forecasts from Dynamic Econometric Models", Journal of Policy Modeling, 14, 4, 535-560.

Chong, Y. Y., and D. F. Hendry (1986) "Econometric Evaluation of Linear Macroeconomic Models", Review of Economic Studies, 53, 4, 671-690.

Chow, G. C. (1960) "Tests of Equality Between Sets of Coefficients in Two Linear Regressions", Econometrica, 28, 3, 591-605.

Clements, M. P., and D. F. Hendry (1993) "On the Limitations of Comparing Mean Square Forecast Errors", Journal of Forecasting, 12, 8, 617-637 (with discussion and reply).

Clements, M. P., and D. F. Hendry (1998) Forecasting Economic Time Series, Cambridge University Press, Cambridge, England.

Clements, M. P., and D. F. Hendry (1999) Forecasting Non-stationary Economic Time Series, MIT Press, Cambridge, Massachusetts.

Clements, M. P., and D. F. Hendry (2000) "Forecasting with Difference-stationary and Trend-stationary Models", Econometrics Journal, 4, 1, S1-S19.

Doornik, J. A., and D. F. Hendry (1996) PcGive Professional 9.0 for Windows, International Thomson Business Press, London.

Engle, R. F., and D. F. Hendry (1993) "Testing Super Exogeneity and Invariance in Regression Models", Journal of Econometrics, 56, 1/2, 119-139.

Ericsson, N. R. (1992a) "Cointegration, Exogeneity, and Policy Analysis: An Overview", Journal of Policy Modeling, 14, 3, 251-280. 
Ericsson, N. R. (1992b) "Parameter Constancy, Mean Square Forecast Errors, and Measuring Forecast Performance: An Exposition, Extensions, and Illustration", Journal of Policy Modeling, 14, 4, 465-495.

Ericsson, N. R., D. F. Hendry, and K. M. Prestwich (1998) "The Demand for Broad Money in the United Kingdom, 1878-1993", Scandinavian Journal of Economics, 100, 1, 289-324 (with discussion).

Ericsson, N. R., and J. S. Irons (1995) "The Lucas Critique in Practice: Theory Without Measurement", Chapter 8 in K. D. Hoover (ed.) Macroeconometrics: Developments, Tensions, and Prospects, Kluwer Academic Publishers, Boston, Massachusetts, 263-312 (with discussion).

Ericsson, N. R., and J. Marquez (1993) "Encompassing the Forecasts of U.S. Trade Balance Models", Review of Economics and Statistics, 75, 1, 19-31.

Ericsson, N. R., and J. Marquez (1998) "A Framework for Economic Forecasting", Econometrics Journal, 1, 1, C228-C266.

Fisher, R. A. (1922) "The Goodness of Fit of Regression Formulae, and the Distribution of Regression Coefficients", Journal of the Royal Statistical Society, 85, 4, $597-612$.

Goldfeld, S. M. (1973) "The Demand for Money Revisited", Brookings Papers on Economic Activity, 1973, 3, 577-638 (with discussion).

Goldfeld, S. M. (1976) "The Case of the Missing Money", Brookings Papers on Economic Activity, 1976, 3, 683-730 (with discussion).

Granger, C. W. J. (1969) "Investigating Causal Relations by Econometric Models and Cross-spectral Methods", Econometrica, 37, 3, 424-438.

Granger, C. W. J. (1989) Forecasting in Business and Economics, Academic Press, Boston, Massachusetts, Second Edition.

Hansen, B. E. (1992a) "Testing for Parameter Instability in Linear Models", Journal of Policy Modeling, 14, 4, 517-533.

Hansen, B. E. (1992b) "Tests for Parameter Instability in Regressions with I(1) Processes", Journal of Business and Economic Statistics, 10, 3, 321-335.

Henderson, J. M., and R. E. Quandt (1971) Microeconomic Theory: A Mathematical Approach, McGraw-Hill, New York.

Hendry, D. F. (1988) "The Encompassing Implications of Feedback versus Feedforward Mechanisms in Econometrics", Oxford Economic Papers, 40, 1, 132-149.

Hendry, D. F. (1995) Dynamic Econometrics, Oxford University Press, Oxford. 
Hendry, D. F. (2000) "Forecast Failure, Expectations Formation, and the Lucas Critique", mimeo, Nuffield College, Oxford, March.

Hendry, D. F., and N. R. Ericsson (eds.) (2001) Understanding Economic Forecasts, forthcoming.

Hendry, D. F., and G. E. Mizon (1999) "The Pervasiveness of Granger Causality in Econometrics", Chapter 5 in R. F. Engle and H. White (eds.) Cointegration, Causality, and Forecasting: A Festschrift in Honour of Clive W. J. Granger, Oxford University Press, Oxford, 102-134.

Hendry, D. F., A. Pagan, and J. D. Sargan (1984) "Dynamic Specification", Chapter 18 in Z. Griliches and M. D. Intriligator (eds.) Handbook of Econometrics, Volume 2, North-Holland, Amsterdam, 1023-1100.

Hoque, A., J. R. Magnus, and B. Pesaran (1988) "The Exact Multi-period Meansquare Forecast Error for the First-order Autoregressive Model", Journal of Econometrics, 39, 3, 327-346.

Judd, J. P., and J. L. Scadding (1982) "The Search for a Stable Money Demand Function: A Survey of the Post-1973 Literature", Journal of Economic Literature, 20, 3, 993-1023.

Lucas, Jr., R. E. (1976) "Econometric Policy Evaluation: A Critique", in K. Brunner and A. H. Meltzer (eds.) The Phillips Curve and Labor Markets, North-Holland, Amsterdam, Carnegie-Rochester Conference Series on Public Policy, Volume 1, Journal of Monetary Economics, Supplement, 19-46 (with discussion).

Marquez, J., and N. R. Ericsson (1993) "Evaluating Forecasts of the U.S. Trade Balance", Chapter 14 in R. C. Bryant, P. Hooper, and C. L. Mann (eds.) Evaluating Policy Regimes: New Research in Empirical Macroeconomics, Brookings Institution, Washington, D.C., 671-732.

Orcutt, G. H., and H. S. Winokur, Jr. (1969) "First Order Autoregression: Inference, Estimation, and Prediction", Econometrica, 37, 1, 1-14.

Schmidt, P. (1974) "The Asymptotic Distribution of Forecasts in the Dynamic Simulation of an Econometric Model", Econometrica, 42, 2, 303-309.

Singer, M. (1997) "Thoughts of a Nonmillenarian", Bulletin of the American Academy of Arts and Sciences, 51, 2, 36-51.

Suits, D. B. (1955) "An Econometric Model of the Watermelon Market", American Journal of Agricultural Economics (formerly the Journal of Farm Economics), 37, $2,237-251$.

Tinbergen, J. (1931) "Ein Schiffbauzyklus?", Weltwirtschaftliches Archiv, 34, 152164 (reprinted as "A Shipbuilding Cycle?" in L. H. Klaassen, L. M. Koyck, and H. J. Witteveen (eds.) (1959) Jan Tinbergen: Selected Papers, North-Holland, Amsterdam, 1-14). 
Wallis, K. F. (1999a) "Asymmetric Density Forecasts of Inflation and the Bank of England's Fan Chart", National Institute Economic Review, 167, January, 106112.

Wallis, K. F. (1999b) "Macroeconometric Modelling", mimeo, Department of Economics, University of Warwick, Coventry, England, August (presented at a conference at the University of Iceland, Reykjavik, Iceland, May 28-29, 1999).

Watson, M. W. (1994) "Vector Autoregressions and Cointegration", Chapter 47 in R. F. Engle and D. L. McFadden (eds.) Handbook of Econometrics, Volume 4, North-Holland, Amsterdam, 2843-2915.

West, K. D. (1996) "Asymptotic Inference About Predictive Ability", Econometrica, $64,5,1067-1084$. 